\title{
Closed-Form Absolute Ruin Problems of the Risk Models with State-Dependent Switched Claims
}

\author{
Yuanxun Liu, Dianli Zhao* \\ College of Science, University of Shanghai for Science and Technology, Shanghai, China \\ Email: ^dianli-zhao@163.com
}

How to cite this paper: Liu, Y.X. and Zhao, D.L. (2017) Closed-Form Absolute Ruin Problems of the Risk Models with State-Dependent Switched Claims. Journal of Applied Mathematics and Physics, 5, 2326-2334. https://doi.org/10.4236/jamp.2017.512190

Received: November 14, 2017

Accepted: December 11, 2017

Published: December 14, 2017

Copyright $\odot 2017$ by authors and Scientific Research Publishing Inc. This work is licensed under the Creative Commons Attribution International License (CC BY 4.0).

http://creativecommons.org/licenses/by/4.0/

\begin{abstract}
This letter mainly investigates a general risk model with the threshold dividend strategy under assumption that the claim amounts obey a state-dependent switched exponential distribution. By establishing the differential-integral equations for the Gerber-Shiu discounted penalty function, and applying the hypergeometric functions, the closed-form absolute ruin probability is derived.
\end{abstract}

\section{Keywords}

Ruin Probability, Threshold Dividend Strategy, Switched Exponential Distribution, Hypergeometric Function

\section{Introduction}

Due to its practical importance, more and more people use the ruin probability, an important leading indicator, to judge whether an insurance company can survive or not. The classical risk model was introduced by Gerber [1]. The function is of the form

$$
U(t)=u_{0}+c t-S(t), S(t)=\sum_{i=0}^{N(t)} X_{i}
$$

where $U(t)$ is the insurance company's surplus at time $t . c$ represents the risk-free rate. $S(t)$ is the total compensation by time $t, X_{i}$ is the i-th compensation; $N(t)$ means the frequency of compensation. $u_{0}$ represents the initial investment for an insurance company. In order to make the classical risk model more realistic, more and more factors have been taken into account. The aggregate premium process taking a linear function of time was considered by Zou, Gao and Xie [2], in which 
the explicit solutions of ruin time were obtained under assumption that the claim amount obeyed exponential distribution. Yao, Yang and Wang [3] studied a dual risk model with fixed transaction cost, and solved the optional control problem by the techniques of quasi-variational inequalities. Yuen, Zhou and Guo [4] discussed the compound Poisson risk model with debit interest and dividend payments, and the explicit expressions for the Gerber-Shiu function and the optimal barrier with exponential claim amounts were given. When the dividend strategy was considered, Yin and Yuen [5] considered a dividend policy aiming to maximize the expected discounted value of dividend until ruin. Taking the threshold dividend strategy into account, two integro-differential equations for the Gerber-Shiu discounted penalty function were obtained by Lin and Pavlova [6]. The ruin model with multiple thresholds was discussed by Lin and Sendova [7]. For more about the related risk model, we can refer to [8] [9] [10] [11] [12] and cited there in. From all the literatures mentioned above, they have a common assumption that the claim sizes obey exponential distribution, i.e. $f(x)=\eta \mathrm{e}^{-\eta x}$. However, in fact, different dividend levels always reflect the difference of the economic environment, which results in the changes of the claim sizes. In detail, when the surplus $u$ is more than the threshold $b$, the claim sizes obey an exponential distribution where the strength is $\eta_{1}$; while $u<b$, the claim sizes obey an exponential distribution with strength $\eta_{2}$. Therefore, the exponential distribution is noted like

$$
f(x)=\eta_{1} \mathrm{e}^{-\eta_{1} x} I_{\{u \geq b\}}+\eta_{2} \mathrm{e}^{-\eta_{2} x} I_{\{u<b\}} .
$$

$I_{\{\}}$is an indicator function, such that $I_{\{u \geq b\}}=1$ and $I_{\{u<b\}}=0$ if $u \geq b$. In order to make it more general, we set the risk model with the claim sizes obeying a switched exponential distribution as follows

$$
f(x)=p \eta_{1} \mathrm{e}^{-\eta_{1} x} I_{\{u \geq b\}}+q \eta_{2} \mathrm{e}^{-\eta_{2} x} I_{\{u<b\}},
$$

where $p$ and $q$ are two suitable positive constants to be chosen later. Clearly, when we choose $p=q=1$ and $\eta_{1}=\eta_{2}$, (3) is the standard exponential distribution applied in [2].

The main aim of this letter is to compute the ruin probability under assumption that the claim sizes obey a switched exponential distribution (3).

The rest of this letter is organized as follows. In Section 2, we firstly get the Gerber-Shiu discounted penalty functions with different surplus. Then, the ruin probability is calculated by assuming the claim sizes obey a switched exponential distribution.

\section{Closed Form Absolute Ruin Probability}

In this letter, we consider the risk model with constant interest rate $r>0$ and debit interest $\rho$. Besides, the studied risk model includes a general threshold dividend strategy. When the surplus is over threshold dividend $b$, we assume the insurance company gets insurance premium at a constant rate $c_{1}$ and earns in- 
terest at a constant rate $r$. If the surplus is between zero and $b$, it will collect insurance premium at a constant rate $c_{2}$ and earns interest at a constant rate $r$. When the surplus is between $-\frac{c_{2}}{\rho}$ and zero, the insurer can borrow an amount of money equal to the deficit at a debit force $\rho$. Meanwhile, the insurer will repay the debts continuously from his premium income. We denote the surplus of the insurer at time $t$ with the credit interest $r$ and debit interest $\rho$ by $U(t)$ which is the solution to

$$
\mathrm{d} U(t)=\left\{\begin{array}{lc}
c_{1} \mathrm{~d} t+r U(t) \mathrm{d} t-\mathrm{d} S(t) ; & u \geq b, \\
c_{2} \mathrm{~d} t+r U(t) \mathrm{d} t-\mathrm{d} S(t) ; & 0 \leq u<b, \\
c_{2} \mathrm{~d} t+\rho U(t) \mathrm{d} t-\mathrm{d} S(t) ; & -\frac{c_{2}}{\rho}<u<0,
\end{array}\right.
$$

where $S(t)=\sum_{i=0}^{N(t)} X_{i}$ is the cumulative damages in time interval $[0, t], c(>0)$ represents the constant rate of premium, $\{N(t), t \geq 0\}$ a Poisson process with intensity $\lambda>0$, which counts the claim numbers in the interval $[0, t]$, and $\left\{X_{i}, i \geq 1\right\} \quad$ (representing the size of claims and independent of $\{N(t), t \geq 0\}$ ) is a sequence of independent and identically distributed nonnegative variables with common distribution function $F(x)$ that satisfies $F(0)=0$ and has a positive mean $\mu=\int_{0}^{\infty} x \mathrm{~d} F(x)$. Let $\left(\Omega, F,\left\{F_{t}\right\}_{t \geq 0}, P\right)$ be a filtered probability space containing all processes and random variables in this letter, satisfying the usual conditions, i.e. $\left\{F_{t}\right\}_{t \geq 0}$ is right continuous and P-complete. In order to obtain the expression of the ruin probability, we should work for two things. First of all, we should get the Gerber-Shiu discounted penalty function. Then, we calculate the ruin probability. So, let's firstly define the Gerber-Shiu discounted penalty function $\varphi(u, b)$.

$$
\varphi(u, b)=E\left\{\mathrm{e}^{-\beta T} W\left(U_{b}\left(T^{-}\right),\left|U_{b}(T)\right|\right) R(T<\infty) \mid U_{b}(0)=u\right\},
$$

where $T$ is the time of bankrupting, $R(T<\infty)$ is an indicator function, which means $R(T<\infty)=1$, if the time of bankrupting is finite value. Else if $R(T<\infty)=0 . W(x, y)$ is a non-negative measurable function satisfying $W(x, y) \in\left(-\frac{c_{2}}{\rho}, \infty\right) \times\left[-\frac{c_{2}}{\rho}, \infty\right) . U_{b}\left(T^{-}\right)$is the surplus immediately before the company bankrupts. Next, we give the integro-differential equations for $\varphi_{i}(u, b)(i=1,2,3)$.

Theorem 2.1. Let $\varphi_{i}(u, b)(i=1,2,3)$ be the Gerber-Shiu discounted penalty function by the surplus $u$ and the threshold $b$ for model(4). Then

$$
\begin{aligned}
\left(r u+c_{1}\right) \varphi_{1}^{\prime}(u, b) & -(\lambda+\beta) \varphi_{1}(u, b)+\lambda \int_{0}^{u-b} \varphi_{1}(u-x, b) \mathrm{d} F(x) \\
& +\lambda \int_{u-b}^{u} \varphi_{2}(u-x, b) \mathrm{d} F(x)+\lambda \int_{u}^{u+\frac{c_{2}}{\rho}} \varphi_{3}(u-x, b) \mathrm{d} F(x) \\
& +\lambda \int_{u+\frac{c_{2}}{\rho}}^{\infty} W(u, x-u) \mathrm{d} F(x)=0,(u \geq b)
\end{aligned}
$$




$$
\begin{aligned}
\left(r u+c_{2}\right) \varphi_{2}^{\prime}(u, b) & -(\lambda+\beta) \varphi_{2}(u, b)+\lambda \int_{0}^{u} \varphi_{2}(u-x, b) \mathrm{d} F(x) \\
& +\lambda \int_{u}^{u+\frac{c_{2}}{\rho}} \varphi_{3}(u-x, b) \mathrm{d} F(x) \\
& +\lambda \int_{u+\frac{c_{2}}{\rho}}^{\infty} W(u, x-u) \mathrm{d} F(x)=0,(0 \leq u<b) ; \\
\left(\rho u+c_{2}\right) \varphi_{3}^{\prime}(u, b) & -(\lambda+\beta) \varphi_{3}(u, b)+\lambda \int_{u}^{u+\frac{c_{2}}{\rho}} \varphi_{3}(u-x, b) \mathrm{d} F(x) \\
& +\lambda \int_{u+\frac{c_{2}}{\rho}}^{\infty} W(u, x-u) \mathrm{d} F(x)=0,\left(-\frac{c_{2}}{\rho}<u<0\right) .
\end{aligned}
$$

the boundary conditions are given like

$$
\begin{gathered}
\varphi_{1}(b, b)=\varphi_{2}\left(b^{-}, b\right), \varphi_{2}(0, b)=\varphi_{3}\left(0^{-}, b\right),\left(r b+c_{2}\right) \varphi_{2}^{\prime}\left(b^{-}, b\right)=\left(r b+c_{1}\right) \varphi_{1}^{\prime}(b, b), \\
\varphi_{3}^{\prime}\left(0^{-}, b\right)=\varphi_{2}^{\prime}(0, b), \varphi_{3}\left(-\frac{c_{2}}{\rho}, b\right)=W\left(-\frac{c_{2}}{\rho}, \frac{c_{2}}{\rho}\right) .
\end{gathered}
$$

Proof. When $u \geq b$, define the cumulative value

$$
h=u \mathrm{e}^{r t}+c_{1} \int_{0}^{t} \mathrm{e}^{r(t-s)} \mathrm{d} s=u \mathrm{e}^{r t}+\frac{c_{1}}{r}\left(\mathrm{e}^{r t}-1\right)
$$

Note that the Gerber-Shiu discounted penalty functions have different expression when the surplus lies within different ranges, we discuss that with the following four cases:

1) When $u_{b}(t) \leq-\frac{c_{2}}{\rho}$, the amount of compensation is $X=h-U_{b}(t)$. Let $\varphi_{3}\left(-\frac{c_{2}}{\rho}, b\right)=1$, its counterpart in the Gerber-Shiu discounted penalty function is $\lambda t \int_{h+\frac{c_{2}}{\rho}}^{\infty} W(h, h-x) \mathrm{d} F(x)$;

2) When $-\frac{c_{2}}{\rho}<u_{b}(t)<0, \lambda t \int_{h}^{h+\frac{c_{2}}{\rho}} \varphi_{3}(h-x, b) \mathrm{d} F(x)$;

3) When $0 \leq u_{b}(t)<b, \lambda t \int_{h-b}^{h} \varphi_{2}(h-x, b) \mathrm{d} F(x)$;

4) When $u_{b}(t) \geq b, \lambda t \int_{0}^{h-b} \varphi_{1}(h-x, b) \mathrm{d} F(x)$.

Therefore, the Gerber-Shiu discounted penalty function is given like,

$$
\begin{aligned}
\varphi_{1}(u, b)= & \lambda t \int_{h+\frac{c_{2}}{\rho}}^{\infty} W(h, h-x) \mathrm{d} F(x)+\lambda t \int_{h}^{h+\frac{c_{2}}{\rho}} \varphi_{3}(h-x, b) \mathrm{d} F(x) \\
& +\lambda t \int_{h-b}^{h} \varphi_{2}(h-x, b) \mathrm{d} F(x)+\lambda t \int_{0}^{h-b} \varphi_{1}(h-x, b) \mathrm{d} F(x) \\
& +(1-\lambda t) \varphi_{1}(h, b)+o(t) .
\end{aligned}
$$

For $\varphi_{1}(h, b)$, by using Taylor expansion, we get

$$
\begin{aligned}
\varphi_{1}(h, b) & =\varphi_{1}(u, b)+\left.\left(\frac{\partial^{2} \varphi_{1}(h, b)}{\partial u^{2}} \frac{\partial^{2} u}{\partial t^{2}}\right)\right|_{(u, b)} t^{2}+\left.\left(\frac{\partial \varphi_{1}(h, b)}{\partial u} \frac{\partial u}{\partial t}\right)\right|_{(u, b)} t+o(t) \\
& =\varphi_{1}(u, b)+\left(r u+c_{1}\right) t \frac{\partial \varphi_{1}(u, b)}{\partial u}+o(t) .
\end{aligned}
$$

Insert (11) into (10) and divide it by $t$. Let $t \rightarrow 0$, we get the desired result. Since 
(7) and (8) can be proved by using the same method, the detail is omitted.

Next, we prove the main result on the absolute ruin probability. For convenience, we denote

$$
\tilde{M}=\left[\begin{array}{cccccc}
0 & 0 & 0 & 0 & L_{5}\left(-\frac{c_{2}}{\rho}\right) & L_{6}\left(-\frac{c_{2}}{\rho}\right) \\
L_{1}(b) & L_{2}(b) & -L_{3}(b) & -L_{4}(b) & 0 & 0 \\
0 & 0 & L_{3}(0) & L_{4}(0) & -L_{5}(0) & -L_{6}(0) \\
-q_{1}(b) & -q_{2}(b) & p_{3}(b) & p_{4}(b) & 0 & 0 \\
0 & 0 & -L_{3}^{\prime}(0) & -L_{4}^{\prime}(0) & L_{5}^{\prime}(0) & L_{6}^{\prime}(0) \\
0 & 0 & 0 & 0 & L_{5}^{\prime}\left(-\frac{c_{2}}{\rho}\right) & L_{6}^{\prime}\left(-\frac{c_{2}}{\rho}\right)
\end{array}\right],
$$

$p_{i}(u)=\left(r u+c_{2}\right) L_{i}^{\prime}(u),(i=3,4), \quad q_{i}(u)=\left(r u+c_{1}\right) L_{i}^{\prime}(u),(i=1,2)$, $C=\left(C_{3}, C_{4}, C_{5}, C_{6}, C_{7}, C_{8}\right)^{\mathrm{T}}, B=(1,0,0,0,0,0)^{\mathrm{T}}$. The hyper-geometric function used later is introduced as follows

$$
M(a, b, x)=\frac{\Gamma(b)}{\Gamma(b-a) \Gamma(a)} \int_{0}^{1} \mathrm{e}^{x t} t^{a-1}(1-t)^{b-a-1} \mathrm{~d} t, b>a>0,
$$

where the Gamma function is $\Gamma(x)=\int_{0}^{\infty} \mathrm{e}^{-t} t^{(x-1)} \mathrm{d} t$.

In view of (6), let $\beta=0$ and $W(x, y)=1$ in the Gerber-Shiu discounted penalty function $\varphi_{i}(u, b)(i=1,2,3)$, we can get the following theorem on the ruin probability.

Theorem 2.2. In model (4), the closed-form ruin probability $\varphi_{i}(u)(i=1,2,3)$ are given like

$$
\begin{aligned}
\varphi_{1}(u)= & C_{3} \mathrm{e}^{-\eta_{1} u} M\left(\frac{r-\lambda p}{r}, 1-\frac{\lambda}{r}, \frac{\left(r u+c_{1}\right) \eta_{1}}{r}\right) \\
& +C_{4}\left(\frac{\left(r u+c_{1}\right) \eta_{1}}{r}\right)^{\frac{\lambda}{r}} \mathrm{e}^{-\eta_{1} u} M\left(\frac{r+(1-p) \lambda}{r}, 1+\frac{\lambda}{r}, \frac{\left.\left(r u+c_{1}\right) \eta_{1}\right)}{r}\right) ; \\
\varphi_{2}(u)= & C_{5} \mathrm{e}^{-\eta_{2} u} M\left(\frac{r-\lambda q}{r}, 1-\frac{\lambda}{r}, \frac{\left(r u+c_{2}\right) \eta_{2}}{r}\right) \\
& +C_{6}\left(\frac{\left(r u+c_{2}\right) \eta_{2}}{r}\right)^{\frac{\lambda}{r}} \mathrm{e}^{-\eta_{2} u} M\left(\frac{r+(1-q) \lambda}{r}, 1+\frac{\lambda}{r}, \frac{\left(r u+c_{2}\right) \eta_{2}}{r}\right) ; \\
\varphi_{3}(u)= & C_{7} \mathrm{e}^{-\eta_{2} u} M\left(\frac{\rho-\lambda q}{\rho}, 1-\frac{\lambda}{\rho}, \frac{\left(\rho u+c_{2}\right) \eta_{2}}{\rho}\right) \\
+ & C_{8}\left(\frac{\left(\rho u+c_{2}\right) \eta_{2}}{\rho}\right)^{\frac{\lambda}{\rho}} \mathrm{e}^{-\eta_{2} u} M\left(\frac{\rho+(1-q) \lambda}{\rho}, 1+\frac{\lambda}{\rho}, \frac{\left(\rho u+c_{2}\right) \eta_{2}}{\rho}\right) ;
\end{aligned}
$$

where $C=\left(C_{3}, C_{4}, \cdots, C_{8}\right)^{\mathrm{T}}$ is solution of $\tilde{M} C=B$.

Proof. By Theorem 2.1, we get

$$
\begin{aligned}
& \left(r u+c_{1}\right) \varphi_{1}^{\prime \prime}(u, b)+\left(r-\lambda+\left(r u+c_{1}\right) \eta_{1}\right) \varphi_{1}^{\prime}(u, b) \\
& -(1-p) \lambda \eta_{1} \varphi_{1}(u, b)=0, u \geq b
\end{aligned}
$$




$$
\begin{aligned}
& \left(r u+c_{2}\right) \varphi_{2}^{\prime \prime}(u, b)+\left(r-\lambda+\left(r u+c_{2}\right) \eta_{2}\right) \varphi_{2}^{\prime}(u, b) \\
& -(1-q) \lambda \eta_{2} \varphi_{2}(u, b)=0,0 \leq u<b ; \\
& \left(\rho u+c_{2}\right) \varphi_{3}^{\prime \prime}(u, b)+\left(\rho-\lambda+\left(\rho u+c_{2}\right) \eta_{2}\right) \varphi_{3}^{\prime}(u, b) \\
& -(1-q) \lambda \eta_{2} \varphi_{3}(u, b)=0,-\frac{c_{2}}{\rho}<u<0 .
\end{aligned}
$$

The boundary conditions are

$$
\begin{aligned}
& \varphi_{3}\left(-\frac{c_{2}}{\rho}, b\right)=1, \lim _{u \rightarrow \infty} \varphi_{1}(u, b)=0, \varphi_{1}(b, b)=\varphi_{2}\left(b^{-}, b\right), \varphi_{2}(0, b)=\varphi_{3}\left(0^{-}, b\right), \\
& \left(r b+c_{2}\right) \varphi_{2}^{\prime}\left(b^{-}, b\right)=\left(r b+c_{1}\right) \varphi_{1}^{\prime}(b, b), \varphi_{3}^{\prime}\left(0^{-}, b\right)=\varphi_{2}^{\prime}(0, b), \varphi_{3}^{\prime}\left(-\frac{c_{2}}{\rho}, b\right)=0 .
\end{aligned}
$$

In order to obtain the expression of ruin probabilities, we employ the confluent hyper-geometric function. In (16), make auxiliary function $\varphi_{1}(u)=X_{1}(u) \mathrm{e}^{a u}$ where $a$ is a constant to be determined later and then

$$
\begin{aligned}
& \left(r u+c_{1}\right) X_{1}^{\prime \prime}(u)+\left[\left(2 a+\eta_{1}\right)\left(r u+c_{1}\right)+r-\lambda\right] X_{1}^{\prime}(u) \\
& +\left[-(1-p) \lambda \eta_{1}+a^{2}\left(r u+c_{1}\right)+a\left(r-\lambda+\left(r u+c_{1}\right) \eta_{1}\right)\right] X_{1}(u)=0,
\end{aligned}
$$

Define $r u+c_{1}=z$, we have

$$
\begin{aligned}
& z X_{1}^{\prime \prime}\left(\frac{z-c_{1}}{r}\right)+\left(1-\frac{\lambda}{r}+\frac{2 a+\eta_{1}}{r} z\right) X_{1}^{\prime}\left(\frac{z-c_{1}}{r}\right) \\
& +\frac{1}{r^{2}}\left[-(1-p) \lambda \eta_{1}+a(r-\lambda)+\left(a^{2}+a \eta_{1}\right) z\right] X_{1}\left(\frac{z-c_{1}}{r}\right)=0 .
\end{aligned}
$$

By choosing $a=-\eta_{1}$,

$$
z X_{1}^{\prime \prime}\left(\frac{z-c_{1}}{r}\right)+\left(1-\frac{\lambda}{r}-\frac{\eta_{1}}{r} z\right) X_{1}^{\prime}\left(\frac{z-c_{1}}{r}\right)+\left(\frac{\lambda p-r}{r} \cdot \frac{\eta_{1}}{r}\right) X_{1}\left(\frac{z-c_{1}}{r}\right)=0
$$

Define $\frac{\eta_{1}}{r} z=v$, we have by (21)

$$
v X_{1}^{\prime \prime}\left(\frac{r v-\eta_{1} c_{1}}{r \eta_{1}}\right)+\left(1-\frac{\lambda}{r}-v\right) X_{1}^{\prime}\left(\frac{r v-\eta_{1} c_{1}}{r \eta_{1}}\right)+\left(\frac{\lambda p-r}{r}\right) X_{1}\left(\frac{r v-\eta_{1} c_{1}}{r \eta_{1}}\right)=0
$$

Let $X_{1}\left(\frac{r v-\eta_{1} c_{1}}{r \eta_{1}}\right)=Y_{1}(v)$, (22) can be rewritten as

$$
v Y_{1}^{\prime \prime}(v)+\left(1-\frac{\lambda}{r}-v\right) Y_{1}^{\prime}(v)-\left(\frac{r-\lambda p}{r}\right) Y_{1}(v)=0,
$$

By using the first kind of confluent hypergeometric function, we obtain that

$$
Y_{1}(v)=C_{3} M\left(\frac{r-\lambda p}{r}, 1-\frac{\lambda}{r} ; v\right)+C_{4}(v)^{\frac{\lambda}{r}} M\left(\frac{r+(1-p) \lambda}{r}, 1+\frac{\lambda}{r} ; v\right),
$$

where $C_{3}$ and $C_{4}$ are constants. Without extra claims, $C_{i}$ are constants for $i \geq 3$ in the following.

Next, the same method can be used to calculate (17) and (18). In (17), set $\varphi_{2}(u)=X_{2}(u) \mathrm{e}^{a u}, r u+c_{2}=z, a=-\eta_{2}, \frac{\eta_{2}}{r} z=v$ and $X_{2}\left(\frac{r v-\eta_{2} c_{2}}{r \eta_{2}}\right)=Y_{2}(v)$, 
then we have

$$
v Y_{2}^{\prime \prime}(v)+\left(1-\frac{\lambda}{r}-v\right) Y_{2}^{\prime}(v)-\left(\frac{r-\lambda q}{r}\right) Y_{2}(v)=0,
$$

The solution of (25) has the form

$$
Y_{2}(v)=C_{5} M\left(\frac{r-\lambda q}{r}, 1-\frac{\lambda}{r} ; v\right)+C_{6}(v)^{\frac{\lambda}{r}} M\left(\frac{r+(1-q) \lambda}{r}, 1+\frac{\lambda}{r} ; v\right) .
$$

Similarly, the solution of (18) is

$$
Y_{3}(v)=C_{7} M\left(\frac{\rho-\lambda q}{\rho}, 1-\frac{\lambda}{\rho} ; v\right)+C_{8}(v)^{\frac{\lambda}{\rho}} M\left(\frac{\rho+(1-q) \lambda}{\rho}, 1+\frac{\lambda}{\rho} ; v\right),
$$

In the sequel, we turn to compute the expression of ruin probability. Inserting $v=\frac{\eta_{1}}{r} z$ and $X_{1}\left(\frac{r v-\eta_{1} c_{1}}{r \eta_{1}}\right)=Y_{1}(v)$ into (24) yields that

$$
\begin{aligned}
X_{1}\left(\frac{z-c_{1}}{r}\right)= & C_{3} M\left(\frac{r-\lambda p}{r}, 1-\frac{\lambda}{r} ; \frac{\eta_{1} z}{r}\right) \\
& +C_{4}\left(\frac{\eta_{1} z}{r}\right)^{\frac{\lambda}{r}} M\left(\frac{r+(1-p) \lambda}{r}, 1+\frac{\lambda}{r} ; \frac{\eta_{1} z}{r}\right) .
\end{aligned}
$$

Denote $r u+c_{1}=z$, then (28) yields that

$$
\begin{aligned}
X_{1}(u)= & C_{3} M\left(\frac{r-\lambda p}{r}, 1-\frac{\lambda}{r} ; \frac{\left(r u+c_{1}\right) \eta_{1}}{r}\right) \\
& +C_{4}\left(\frac{\left(r u+c_{1}\right) \eta_{1}}{r}\right)^{\frac{\lambda}{r}} M\left(\frac{r+(1-p) \lambda}{r}, 1+\frac{\lambda}{r} ; \frac{\left(r u+c_{1}\right) \eta_{1}}{r}\right) .
\end{aligned}
$$

Define $\varphi_{1}(u)=X_{1}(u) \mathrm{e}^{-\eta_{1} u}$, we have by (29)

$$
\begin{aligned}
\varphi_{1}(u)= & C_{3} \mathrm{e}^{-\eta_{1} u} M\left(\frac{r-\lambda p}{r}, 1-\frac{\lambda}{r} ; \frac{\left(r u+c_{1}\right) \eta_{1}}{r}\right) \\
& +C_{4}\left(\frac{\left(r u+c_{1}\right) \eta_{1}}{r}\right)^{\frac{\lambda}{r}} \mathrm{e}^{-\eta_{1} u} M\left(\frac{r+(1-p) \lambda}{r}, 1+\frac{\lambda}{r} ; \frac{\left(r u+c_{1}\right) \eta_{1}}{r}\right) .
\end{aligned}
$$

Then we can use the same method to solve (26) and (27) like that

$$
\begin{aligned}
\varphi_{2}(u)= & C_{5} \mathrm{e}^{-\eta_{2} u} M\left(\frac{r-\lambda q}{r}, 1-\frac{\lambda}{r}, \frac{\left(r u+c_{2}\right) \eta_{2}}{r}\right) \\
& +C_{6}\left(\frac{\left(r u+c_{2}\right) \eta_{2}}{r}\right)^{\frac{\lambda}{r}} \mathrm{e}^{-\eta_{2} u} M\left(\frac{r+(1-q) \lambda}{r}, 1+\frac{\lambda}{r}, \frac{\left(r u+c_{2}\right) \eta_{2}}{r}\right) ; \\
\varphi_{3}(u)= & C_{7} \mathrm{e}^{-\eta_{2} u} M\left(\frac{\rho-\lambda q}{\rho}, 1-\frac{\lambda}{\rho}, \frac{\left(\rho u+c_{2}\right) \eta_{2}}{\rho}\right) \\
& +C_{8}\left(\frac{\left(\rho u+c_{2}\right) \eta_{2}}{\rho}\right)^{\frac{\lambda}{\rho}} \mathrm{e}^{-\eta_{2} u} M\left(\frac{\rho+(1-q) \lambda}{\rho}, 1+\frac{\lambda}{\rho}, \frac{\left(\rho u+c_{2}\right) \eta_{2}}{\rho}\right) .
\end{aligned}
$$

To calculate the coefficients of the ruin probability $C=\left(C_{3}, C_{4}, \cdots, C_{8}\right)^{\mathrm{T}}$, we firstly 
rewrite (30)-(32) to be

$$
\begin{aligned}
& \varphi_{1}(u, b)=C_{3} L_{1}(u)+C_{4} L_{2}(u) ; \varphi_{2}(u, b)=C_{5} L_{3}(u)+C_{6} L_{4}(u) \\
& \text { and } \varphi_{3}(u, b)=C_{7} L_{5}(u)+C_{8} L_{6}(u) .
\end{aligned}
$$

By inserting boundary conditions into (30), (31) and (32), we get

$$
\left\{\begin{array}{l}
C_{7} L_{5}\left(-\frac{c_{2}}{\rho}\right)+C_{8} L_{6}\left(-\frac{c_{2}}{\rho}\right)=1 ; C_{3} L_{1}(b)+C_{4} L_{2}(b)-C_{5} L_{3}(b)-C_{6} L_{4}(b)=0 ; \\
C_{5} L_{3}(0)+C_{6} L_{4}(0)-C_{7} L_{5}(0)-C_{8} L_{6}(0)=0 ; \\
\left(r b+c_{2}\right) C_{5} L_{3}^{\prime}(b)+\left(r b+c_{2}\right) C_{6} L_{4}^{\prime}(b)-\left(r b+c_{1}\right) C_{3} L_{1}^{\prime}(b)-\left(r b+c_{1}\right) C_{4} L_{2}^{\prime}(b)=0 ; \\
C_{7} L_{5}^{\prime}(0)+C_{8} L_{6}^{\prime}(0)-C_{5} L_{3}^{\prime}(0)-C_{6} L_{4}^{\prime}(0)=0 ; \\
C_{7} L_{5}^{\prime}\left(-\frac{c_{2}}{\rho}\right)+C_{8} L_{6}^{\prime}\left(-\frac{c_{2}}{\rho}\right)=0,
\end{array}\right.
$$

which can be simplified as $\tilde{M} C=B$. The proof is complete.

Remark 1. Let $p=q=1$ and $\eta_{1}=\eta_{2}$, Theorem 2.1 reduces to the related results given in [2]. In this sense, the previously-known results are generalized. Further, Theorem 2.2 shows us the closed-form solution of the ruin probability which is valuable in theoretical applications.

\section{Conclusion}

In this letter, we discuss the ruin probability of the risk model under assumption that the claim amounts obey the switched exponential distribution. The closed-form expressions of the ruin probability are obtained by using the first kind of hypergeometric function. The obtained results may give us guidance in facing up to the economic crisis.

\section{References}

[1] Gerber, H.U. (1981) On the Probability of Ruin in the Presence of a Linear Dividend Barrier. Scandinavian Actuarial Journal, 1981, 105-115. https://doi.org/10.1080/03461238.1981.10413735

[2] Zou, W., Gao, J. and Xie, J. (2014) On the Expected Discounted Penalty Function and Optimal Dividend Strategy for a Risk Model with Random Incomes and Interclaim-Dependent Claim Sizes. Journal of Computational and Applied Mathematics, 255, 270-281. https://doi.org/10.1016/j.cam.2013.05.004

[3] Yao, D., Yang, H. and Wang, R. (2011) Optimal Dividend and Capital Injection Problem in the Dual Model with Proportional and Fixed Transaction Costs. European Journal of Operational Research, 211, 568-576. https://doi.org/10.1016/j.ejor.2011.01.015

[4] Yuen, K., Zhou, M. and Guo, J. (2008) On a Risk Model with Debit Interest and Dividend Payments. Statistics and Probability Letters, 78, 2426-2432. https://doi.org/10.1016/j.spl.2008.02.021

[5] Yin, C. and Yuen, K. (2011) Optimality of the Threshold Dividend Strategy for the Compound Poisson Model. Statistics and Probability Letters, 81, 1841-1846. https://doi.org/10.1016/j.spl.2011.07.022

[6] Lin, X. and Pavlova, K.P. (2006) The Compound Poisson Risk Model with a Thre- 
shold Dividend Strategy. Insurance: Mathematics and Economics, 38, 57-80. https://doi.org/10.1016/j.insmatheco.2005.08.001

[7] Lin, X. and Sendova, K.P. (2008) The Compound Poisson Risk Model with Multiple Thresholds. Insurance: Mathematics and Economics, 42, 617-627. https://doi.org/10.1016/j.insmatheco.2007.06.008

[8] Fu, K.-A. (2016) On Joint Ruin Probability for a Bidimensional Levy-Driven Risk Model with Stochastic Returns and Heavy-Tailed Claims. Journal of Mathematical Analysis and Applications, 442, 17-30. https://doi.org/10.1016/j.jmaa.2016.04.042

[9] Zhao, Y. and Yao, J. (2005) Actuarial Module of Enterprise-Complemented Pension Scheme While Taking Vasicek as Stochastic Interest Rate Module. Journal of University of Shanghai for Science and Technology, 27, 268-270. (In Chinese)

[10] Wang, Q., Xu, Z. and Gao, Y. (2009) Research on the Pricing Problem of Whole-LifeEquity-Linked Policies. Journal of University of Shanghai for Science and Technology, 31, 80-84. (In Chinese)

[11] Chadjiconstantinidis, S. and Papaioannou, A.D. (2013) On a Perturbed by Diffusion Compound Poisson Risk Model with Delayed Claims and Multi-Layer Dividend Strategy. Journal of Computational and Applied Mathematics, 253, 26-50. https://doi.org/10.1016/j.cam.2013.02.014

[12] Lu, D. and Zhang, B. (2016) Some Asymptotic Results of the Ruin Probabilities in a Two-Dimensional Renewal Risk Model with Some Strongly Subexponential Claims. Statistics and Probability Letters, 114, 20-29.

https://doi.org/10.1016/j.spl.2016.03.005 